\title{
Exotic molecular states in the decays of vector bottomonia
}

\author{
V. Baru ${ }^{1,2,3}$, E. Epelbaum ${ }^{4}$, A. A. Filin ${ }^{4}$, C. Hanhart ${ }^{5}$, A. V. Nefediev ${ }^{3,6, *}$, and Q. Wang ${ }^{1}$ \\ ${ }^{1}$ Helmholtz-Institut für Strahlen- und Kernphysik and Bethe Center for Theoretical Physics, Universität \\ Bonn, D-53115 Bonn, Germany \\ ${ }^{2}$ Institute for Theoretical and Experimental Physics, B. Cheremushkinskaya 25, 117218 Moscow, Rus- \\ sia \\ ${ }^{3}$ P.N. Lebedev Physical Institute of the Russian Academy of Sciences, 119991, Leninskiy Prospect 53, \\ Moscow, Russia \\ ${ }^{4}$ Ruhr University Bochum, Faculty of Physics and Astronomy, Institute for Theoretical Physics II, D- \\ 44780 Bochum, Germany \\ ${ }^{5}$ Forschungszentrum Jülich, Institute for Advanced Simulation, Institut für Kernphysik and Jülich Cen- \\ ter for Hadron Physics, D-52425 Jülich, Germany \\ ${ }^{6}$ National Research Nuclear University MEPhI, 115409, Kashirskoe highway 31, Moscow, Russia
}

\begin{abstract}
The most recent experimental data for the decays of the vector bottomonium $\Upsilon(10860)$ proceeding through the formation of the states $Z_{b}(10610)$ and $Z_{b}(10650)$ are analysed simultaneously using solutions of the LippmannSchwinger equations which respect constraints from unitarity and analyticity. The interaction potential in the open-bottom channels $B^{(*)} \bar{B}^{*}$ contains shortrange interactions as well as the one-pion exchange; both types of the interaction are taken into account fully nonperturbatively. This way, all parameters of the interaction are fixed directly from the data and the pole positions for the $Z_{b}$ 's are determined as a prediction. In particular, both $Z_{b}$ states are found to be described by resonance poles located on the unphysical Riemann sheets in the vicinity of the corresponding thresholds. The heavy quark spin symmetry (HQSS) is employed to predict, in a parameter-free way, the pole positions and the line shapes in the elastic and inelastic channels for the $Z_{b}$ s' spin partner states $W_{b J}$ with the quantum numbers $J^{++}(J=0,1,2)$. Such spin partners can be produced in radiative decays of the vector bottomonium $\Upsilon(10860)$ and are expected to be detected in the Belle-II experiment.
\end{abstract}

\section{Introduction}

In the last fifteen years many states in the spectrum of charmonium and bottomonium were discovered experimentally, and while some of them can be well described by the quark model, others can not. Hadrons which demonstrate properties at odds with the quark model are usually called exotic. Most of exotic hadrons reside in the vicinity of strong open-flavour thresholds and this leaves a strong footprint on their properties. The first discovered and the best studied to date state of this type is the $X(3872)$ which lies extremely close to the neutral $D \bar{D}^{*}$ threshold. The $X(3872)$ was discovered by the Belle Collaboration in 2003 [1]. The unusual nature of this state was reflected in the name it was given - the $X$. The discovery

\footnotetext{
*e-mail: nefediev@ lebedev.ru
} 

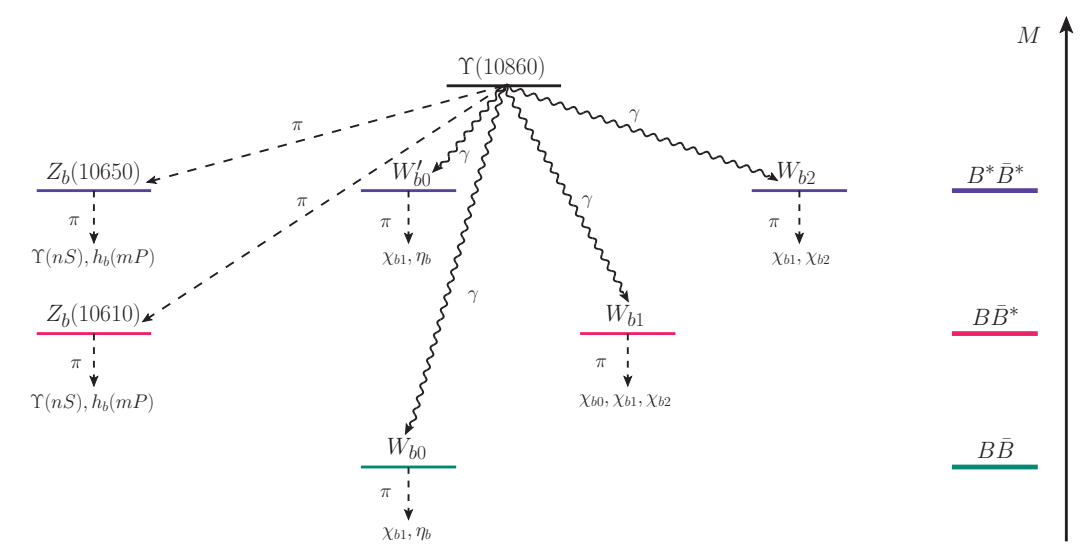

Figure 1. Studied here decays of the $\Upsilon(10860)$ proceeding through the formation of the $Z_{b}$ 's and $W_{b J}$ 's.

of the $X(3872)$ started a new era in the heavy-quark spectroscopy with an important role played by the $B$-factories at $e^{+} e^{-}$colliders, such as Belle at KEK and BABAR at SLAC. Further studies revealed more surprises. Not only isoscalar exotic states were observed but, in addition, several charged resonances were found which, at the same time, unavoidably contained a heavy quark-antiquark pair. Since the minimal quark content of such charged states is four-quark they must have exotic nature. In particular, charged $1^{+-}$states $Z_{b}(10610)$ and $Z_{b}(10650)$ in the spectrum of bottomonium were observed by the Belle Collaboration in 2011 [2]. Parameters of these states $\left(M_{Z_{b}}=10607.2 \pm 2.0 \mathrm{MeV}, \Gamma_{Z_{b}}=18.4 \pm 2.4 \mathrm{MeV}\right.$, $M_{Z_{b}^{\prime}}=10652.2 \pm 1.5 \mathrm{MeV}, \Gamma_{Z_{b}^{\prime}}=11.5 \pm 2.2 \mathrm{MeV}$ [3]) demonstrate that they reside close to the $B \bar{B}^{*}$ and $B^{*} \bar{B}^{*}$ threshold, respectively. The seven observation modes for these states are

$$
\Upsilon(10860) \rightarrow \pi Z_{b}^{(\prime)} \rightarrow \pi B \bar{B}^{*}, \pi B^{*} \bar{B}^{*}, \pi \pi \Upsilon(1 S), \pi \pi \Upsilon(2 S), \pi \pi \Upsilon(3 S), \pi \pi h_{b}(1 P), \pi \pi h_{b}(2 P),
$$

The molecular interpretation of the $Z_{b}$ 's, suggested in the pioneering work [2] and further developed in many later works allows one to explain the nearly equal rates of the two-pion decays $\Upsilon(10860) \rightarrow \pi \pi h_{b}$ and $\Upsilon(10860) \rightarrow \pi \pi \Upsilon$ which proceed with or without the heavy quark spin flip, that, naively, would imply a strong heavy-quark spin symmetry (HQSS) violation, which is quite unexpected and unnatural for bottomonia. Furthermore, the line shapes of the $Z_{b}$ 's in the reactions quoted in Eq. (1) can be well described in the molecular model [4-7].

Hadronic molecules are characterised by a large probability to find a two-hadron component in their wave function (see the review [8] and references therein). Obviously, proximity of open-flavour thresholds implies a large admixture of the meson-meson component in the wave function of the resonance, so that it becomes a strong candidate for a molecular state. Meanwhile, it remains an open question whether the resonance is a bound or virtual state, or it can be described as an above-threshold resonance or a coupled-channel pole. Binding forces responsible for the formation of the resonance can also differ from model to model or they can be treated in a model-independent way using an effective field theory (EFT) framework, as we do in this work. Then the free parameters, such as the low-energy constants and various couplings, should be fixed from a combined analysis of the existing experimental data - see, for example, Ref. [7].

Since the degrees of freedom related to the spin of the heavy quark are strongly suppressed, HQSS predicts the existence of spin partners of the $Z_{b}$ 's with a different heavy-quark spin orientation and with the quantum numbers $J^{P C}=J^{++}(J=0,1,2)[2,9,10]$. Because 
of a different $C$-parity these states cannot be produced in pionic transitions from the vector bottomonium $\Upsilon(10860)$ but should be seen in its radiative decays. The scheme of decays of $\Upsilon(10860)$ to various final states which proceed through the formation of the $Z_{b}$ 's and $W_{b J}$ 's at the intermediate stage is shown in Fig. 1.

\section{Building a common EFT for the $Z_{b}$ 's and $W_{b J}$ 's}

Effective field theory allows one to construct a model-independent and systematically improvable approach to the system at hand. To this end one needs to build a potential which is (i) consistent with all relevant symmetries of the system and (ii) includes all relevant interactions up to the given order in the chiral expansion, that is in the expansion in the parameter $\chi=Q / \Lambda_{h}$, where $\Lambda_{h}$ is a hard scale of the problem while $Q$ represents soft scales. Then any physical quantity of interest ' $X$ ' calculated to the given order $v$ can be represented as a series in powers of $\chi$ and the theoretical uncertainty comes from the neglected term of the order $v+1$

$$
X^{(v)}(Q)=\sum_{n=0}^{v} \alpha_{n} \chi^{n}, \quad \delta X=X^{(v+1)}-X^{(v)}=\alpha_{v+1} \chi^{v+1} .
$$

The EFT able to describe the $Z_{b}$ states together with their spin partners $W_{b J}$ should respect HQSS and treat the scales of the order $p_{\text {typ }}=\sqrt{m_{B} \delta} \simeq 500 \mathrm{MeV}$, where $\delta=m_{B^{*}}-m_{B} \approx$ $45 \mathrm{MeV}$, as soft scales in order to be able to cover a sufficiently wide energy range from the $B \bar{B}$ to $B^{*} \bar{B}^{*}$ threshold. It has to be noticed that while a high accuracy of HQSS in the $b$-quark sector is guaranteed by a very large mass of the $b$-quark, $\Lambda_{\mathrm{QCD}} / m_{b} \ll 1$, the situation with the chiral expansion of the potential is more subtle. Indeed, for the standard value of $\Lambda_{h} \simeq 1 \mathrm{GeV}$ the parameter $\chi \simeq 1 / 2$, so that convergence of the EFT series needs to be a special concern. A large value of the soft scale $Q \simeq p_{\text {typ }}$ implies that all interactions which generate scales below or comparable with $Q$ have to be included explicitly. In particular, the one-pion exchange (OPE) and one- $\eta$ exchange (OEE) should be treated this way. Meanwhile, it was found in Ref. [7] that the impact of the OEE on observables is tiny, so it will not be considered here.

The OPE potential contributes not only to $S$-wave interactions but also to higher waves, in particular, to the $D$-wave one. Given the large momenta generated by the splitting of the relevant thresholds, the importance of such $D$ waves may be quite high. Also, following work [7], we promote the $S$-to- $D O\left(p^{2}\right)$ contact term (CT) from NLO to LO, that improves renormalisability of the theory and, in addition, we include the $S$-to- $S O\left(p^{2}\right)$ contact term explicitly, that is, we build an almost complete NLO, up to corrections from the long-range two-pion exchange (TPE) which does not follow from the iterations of the OPE (that is, crossbox diagrams and so on). It was demonstrated in Ref. [7] that the long-range part of the OPE provides a visible but moderate effect on observables, so that the impact of the long-range TPE on observables is expected to be quite small. We disregard these contributions.

\section{Data analysis for the $Z_{b}$ 's}

The coupled-channel problem is formulated for the set of elastic $B^{(*)} \bar{B}^{(*)}$ channels (in what follows labelled by greek letters $\alpha, \beta, \ldots$ ),

$$
\begin{aligned}
& 1^{+-}: B \bar{B}^{*}\left({ }^{3} S_{1},-\right), B^{*} \bar{B}^{*}\left({ }^{3} S_{1}\right), B \bar{B}^{*}\left({ }^{3} D_{1},-\right), B^{*} \bar{B}^{*}\left({ }^{3} D_{1}\right), \\
& 0^{++}: B \bar{B}\left({ }^{1} S_{0}\right), B^{*} \bar{B}^{*}\left({ }^{1} S_{0}\right), B^{*} \bar{B}^{*}\left({ }^{5} D_{0}\right), \\
& 1^{++}: B \bar{B}^{*}\left({ }^{3} S_{1},+\right), B \bar{B}^{*}\left({ }^{3} D_{1},+\right), B^{*} \bar{B}^{*}\left({ }^{5} D_{1}\right), \\
& 2^{++}: B^{*} \bar{B}^{*}\left({ }^{5} S_{2}\right), B \bar{B}\left({ }^{1} D_{2}\right), B \bar{B}^{*}\left({ }^{3} D_{2}\right), B^{*} \bar{B}^{*}\left({ }^{1} D_{2}\right), B^{*} \bar{B}^{*}\left({ }^{5} D_{2}\right), B^{*} \bar{B}^{*}\left({ }^{5} G_{2}\right),
\end{aligned}
$$



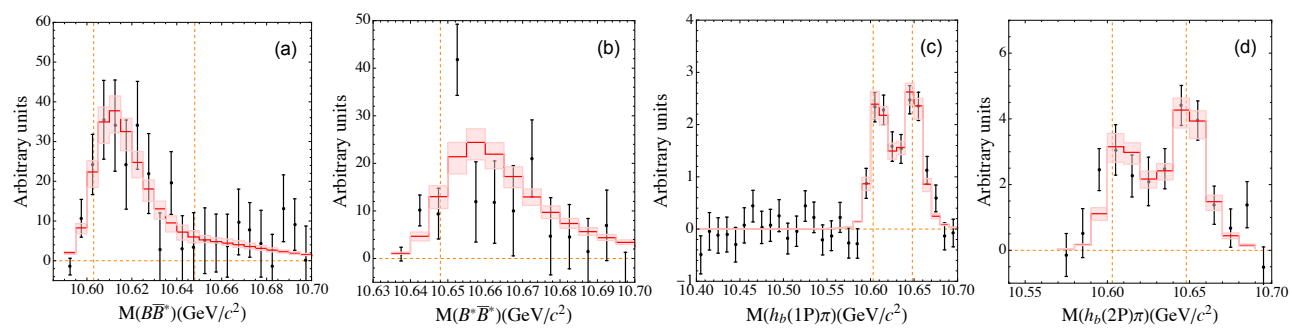

Figure 2. The line shapes of the $Z_{b}$ 's in the elastic and inelastic channels: the experimental data are given by the black dots with error bars while the theoretical fit with the $1 \sigma$ uncertainty interval due to the experimental error is shown by the red histogram. The experimental data are from Refs. [13, 14].

where a particular partial wave and (if necessary) the $C$-parity of the system are quoted explicitly in parenthesis. It is important to emphasise that at leading order only $S$-wave states are coupled to each other through the short-range interactions while $D$ and even $G$ waves get coupled as soon as the OPE is involved. The importance of retaining all relevant partial waves for the renormalisability of the resulting theory was discussed in detail in Ref. [11]. Following the logic of Refs. [5-7, 12] we neglect direct interactions between the inelastic channels, so that they decouple from the system of coupled-channel equations providing only an additional effective potential between elastic channels, that is, schematically, $V_{\mathrm{el}-\mathrm{el}}^{\mathrm{eff}}=V_{\mathrm{el}-\mathrm{el}}^{\mathrm{CT}}+V_{\mathrm{el}-\mathrm{el}}^{\mathrm{OPE}}+\sum_{\mathrm{inel}} V_{\mathrm{el}-\text {-inel-el }}$. Then the Lippmann-Schwinger equation formulated for the elastic off-shell amplitude $T\left(M, \boldsymbol{p}, \boldsymbol{p}^{\prime}\right)$ reads

$$
T_{\alpha \beta}\left(M, \boldsymbol{p}, \boldsymbol{p}^{\prime}\right)=V_{\alpha \beta}^{\mathrm{eff}}\left(\boldsymbol{p}, \boldsymbol{p}^{\prime}\right)-\sum_{\gamma} \int \frac{d^{3} q}{(2 \pi)^{3}} V_{\alpha \gamma}^{\mathrm{eff}}(\boldsymbol{p}, \boldsymbol{q}) G_{\gamma}(M, \boldsymbol{q}) T_{\gamma \beta}\left(M, \boldsymbol{q}, \boldsymbol{p}^{\prime}\right),
$$

where $G_{\gamma}(M, q)$ is the two-body Green's function of the $B^{(*)} \bar{B}^{(*)}$ pair. Equation (3) is solved numerically and the parameters of the theory (the contact interactions and the coupling constants responsible for the transitions between various channels) are extracted directly from a combined fit to the data for the $Z_{b}(10610)$ and $Z_{b}(10650)$ states — see Fig. 2 and Refs. [7, 12].

The conclusions deduced from the fit obtained can be summarised as follows:

- The description of the data is very good $\left(\chi^{2} /\right.$ d.o.f $\left.=0.83\right)$.

- The existing data are compatible with HQSS.

- The effect from the (long range) pion exchange is visible.

- $B \bar{B}^{*}-B^{*} \bar{B}^{*}$ transitions are enhanced by the pions but tamed by the $S$-to- $D$ contact terms.

\section{Predictions for the $W_{b J}$ 's}

The EFT approach built in Ref. [12], with all the parameters fixed directly to the data for the $Z_{b}$ 's, allows one to make parameter-free predictions for their spin partners $W_{b J}$ 's. The corresponding line shapes are shown in Fig. 3 and the pole positions are quoted in Table 4. In particular, we find that all $Z_{b}$ and $W_{b J}$ states are above-threshold resonances, that stresses the importance of pions since only a nontrivial interplay of different dynamics may allow one to move the poles responsible for the physical state to such a position - otherwise, that is, in the pionless theory, all above states appear to be virtual states - see a detailed discussion 


\begin{tabular}{cllccc}
$J^{P C}$ & State & Threshold & Signature & $E_{\text {pole }}$ w.r.t. threshold $[\mathrm{MeV}]$ & Residue at $E_{\text {pole }}$ \\
\hline $1^{+-}$ & $Z_{b}$ & $B \bar{B}^{*}$ &,,,--+ & $(-2.3 \pm 0.5)-i(1.1 \pm 0.1)$ & $(-1.2 \pm 0.2)+i(0.3 \pm 0.2)$ \\
$1^{+-}$ & $Z_{b}^{\prime}$ & $B^{*} \bar{B}^{*}$ &,,--- & $(1.8 \pm 2.0)-i(13.6 \pm 3.1)$ & $(1.5 \pm 0.2)-i(0.6 \pm 0.3)$ \\
$0^{++}$ & $W_{b 0}$ & $B \bar{B}$ &,,,--+ & $(2.3 \pm 4.2)-i(16.0 \pm 2.6)$ & $(1.7 \pm 0.6)-i(1.7 \pm 0.5)$ \\
$0^{++}$ & $W_{b 0}^{\prime}$ & $B^{*} \bar{B}^{*}$ &,,,--- & $(-1.3 \pm 0.4)-i(1.7 \pm 0.5)$ & $(-0.9 \pm 0.3)-i(0.3 \pm 0.2)$ \\
$1^{++}$ & $W_{b 1}$ & $B \bar{B}^{*}$ &,,,--+ & $(10.2 \pm 2.5)-i(15.3 \pm 3.2)$ & $(1.3 \pm 0.2)-i(0.4 \pm 0.2)$ \\
$2^{++}$ & $W_{b 2}$ & $B^{*} \bar{B}^{*}$ &,,,---- & $(7.4 \pm 2.8)-i(9.9 \pm 2.2)$ & $(0.7 \pm 0.1)-i(0.3 \pm 0.1)$
\end{tabular}

Table 1. The pole positions and the residues describing the states $Z_{b}$ 's and $W_{b J}$ 's. The fourth column contains the signs of the imaginary parts of the momenta in the effective inelastic channel, $B \bar{B}, B \bar{B}^{*}$, and $B^{*} \bar{B}^{*}$ channel, in order; a missing sign indicates that this channel is uncoupled. Uncertainties correspond to a $1 \sigma$ deviation in the parameters allowed by the fit to the data in the channels with $J^{P C}=1^{+-}$where the $Z_{b}^{(\prime)}$ states reside [7]. For the discussion of the residues quoted in the last column see Ref. [12].

in Ref. [12]. The evaluated partial branchings can be found in Ref. [12]. As an example, we quote several relations which can be deduced from the individual partial branching fractions,

$$
\begin{gathered}
\Gamma_{B \bar{B}^{*}\left({ }^{3} S_{1}\right)}^{1_{+}^{+}}: \Gamma_{B^{*} \bar{B}^{*}\left({ }^{5} S_{2}\right)}^{2^{++}}: \Gamma_{B \bar{B}\left({ }^{1} S_{0}\right)}^{0^{++}}: \Gamma_{B^{*} \bar{B}^{*}\left({ }^{1} S_{0}\right)}^{0_{++}} \approx 15: 12: 5: 1, \\
\Gamma_{B \bar{B}\left({ }^{1} D_{2}\right)}^{2^{++}}: \Gamma_{B \bar{B}^{*}\left({ }^{3} D_{2}\right)}^{2^{++}}: \Gamma_{B^{*} \bar{B}^{*}\left({ }^{1} S_{0}\right)}^{0^{++}} \approx 3: 3: 2 .
\end{gathered}
$$

The uncertainty of the theoretical predictions can be roughly estimated using the method outlined in Eq. (2). Then, since a typical energy scale associated with the line shapes, pole positions and so on is of the order of $E_{\mathrm{typ}} \simeq 10-15 \mathrm{MeV}$, then, as a very conservative estimate, one has $\delta E \simeq E_{\text {typ }} \cdot \chi \simeq 15 \cdot(1 / 2) \simeq 7.5 \mathrm{MeV}$.

\section{Conclusions}

We conclude that the EFT approach to near-threshold molecular states suggested and employed in this work (i) is compatible with all constraints from unitarity, analyticity and HQSS, (ii) incorporates all most relevant types of interactions and scales, (iii) is able to explain the existing data on the $Z_{b}(10610)$ and $Z_{b}(10650)$ and (iv) is suitable to predict in a parameterfree way spin partners $W_{b J}$. These states are expected to be produced in radiative decays from the $\Upsilon(10860)$ (see Fig. 1) and seen at the next generation $B$-factory Belle-II due to its high luminosity and, as a result, due to high statistics available at it.

Further theoretical developments of the approach include a complete NLO calculation to improve theoretical accuracy, inclusion of the two-pion final state interaction in $\Upsilon(10860) \rightarrow$ $\pi \pi \Upsilon(n S)(n=1,2,3)$ channels to improve parameters extraction from data, inclusion of the compact component of the wave function to treat isoscalar molecules, extension to the $S U(3)$ flavour group for light quarks to predict molecules with the strange quark, tests of accuracy of HQSS to better control theoretical uncertainties which is especially important in the $c$-sector.

This work was supported in part by the DFG (Grant No. TRR110) and the NSFC (Grant No. 11621131001) through the funds provided to the Sino-German CRC 110 "Symmetries and the Emergence of Structure in QCD". Work of V.B. and A.N. was supported by the Russian Science Foundation (Grant No. 18-12-00226).

\section{References}

[1] S.K. Choi et al. (Belle), Phys. Rev. Lett. 91, 262001 (2003), hep-ex/0309032 

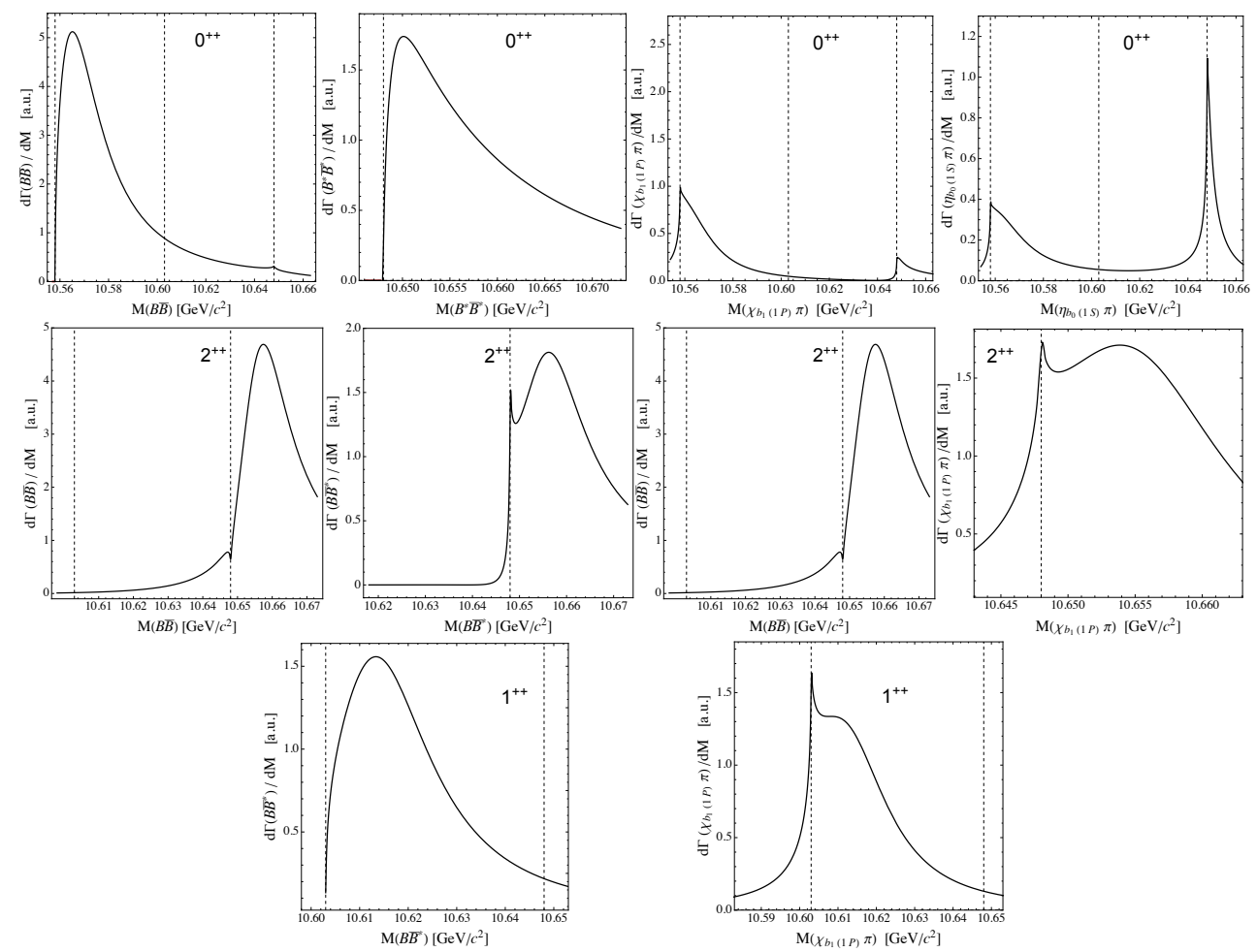

Figure 3. Predictions for the line shapes of the $W_{b J}$ states with $J=0$ (upper row), $J=1$ (lower row) and $J=2$ (middle row) in the elastic and inelastic channels.

[2] A.E. Bondar et al., M.B. Voloshin, Phys. Rev. D84, 054010 (2011), 1105.4473

[3] M. Tanabashi et al. (Particle Data Group), Phys. Rev. D98, 030001 (2018)

[4] T. Mehen, J. Powell, Phys. Rev. D88, 034017 (2013), 1306.5459

[5] C. Hanhart et al., Phys. Rev. Lett. 115, 202001 (2015), 1507.00382

[6] F.-K. Guo et al., Phys. Rev. D93, 074031 (2016), 1602 .00940

[7] Q. Wang et al., Phys. Rev. D98, 074023 (2018), 1805.07453

[8] F. K. Guo, et al., Rev. Mod. Phys. 90, 015004 (2018), 1705.00141

[9] M. B. Voloshin, Phys. Rev. D 84, 031502 (2011), 1105 . 5829

[10] T. Mehen and J. W. Powell, Phys. Rev. D 84, 114013 (2011), 1109. 3479

[11] V. Baru et al., Phys. Lett. B763, 20 (2016), 1605.09649

[12] V. Baru et al., Phys. Rev. D, in press, 1901. 10319

[13] A. Bondar et al. (Belle), Phys. Rev. Lett. 108, 122001 (2012), 1110. 2251

[14] A. Garmash et al. (Belle), Phys. Rev. Lett. 116, 212001 (2016), 1512.07419 